\title{
El papel de los puertos industriales del sur de Tamaulipas en el proceso de globalización de México
}

\section{The role of industrial ports in the south of Tamaulipas in the process of globalization in México}

\author{
Zorrilla González, José (20 Io), LOS PUERTOS DEL SUR DE \\ TAMaUlipas, REALIDADES Y PERSPECTIVAS, El Colegio de Tamaulipas, \\ CiUdad Victoria, 2 I 7 PP., ISBN: I 3 978-607-950 I 2-I-I
}

El papel de los puertos industriales en México ha sido un tema de debate constante, principalmente por el efecto regional que el diseńo y operación de este tipo de infraestructuras estratégicas han producido en los últimos 30 años en México.

El tema que aborda José Zorrilla González en su libro Los puertos del sur de Tamaulipas, realidades y perspectivas, se enmarca en la evaluación de una política pública que en México ha tenido sus altibajos, primero como un motor de desarrollo regional y posteriormente en su política de expansión económica y regional, sobre todo por el giro estratégico que el Estado mexicano le ha dado a la planeación territorial, tanto en los ámbitos regionales como en los urbanos, y no menos importante en el desarrollo del sistema portuario mexicano durante la segunda mitad del siglo xx. El estudio abarca el periodo 1970-2005, lapso donde se analiza la formación y crecimiento del Sistema Portuario Tampico-Altamira, localizado al sur del estado de Tamaulipas, en una etapa de desarrollo basada en la política económica denominada de sustitución de importaciones, y después en un periodo de contracción y crisis financiera de las décadas de los ochenta y noventa, sin dejar de estudiar los efectos que tuvo en la economía nacional por la apertura comercial incipiente y la adhesión al Acuerdo General sobre Aranceles Aduaneros y Comercio (GATT) que se consolida en el marco del Tratado de Libre Comercio de América del Norte (TLCAN) en el año 1992.

Las preguntas de investigación iniciales se centran principalmente en el efecto que ha tenido la apertura comercial de la economía mexicana en el sistema portuario nacional, qué efectos se han producido en el desempeño puntual de los puertos de Tampico-Altamira, su reacomodo administrativo y de operaciones a partir de la reforma a la Ley de Puertos de 1993 y, por último, cómo estos puertos industriales han sido capaces de 
aprovechar las ventajas de desarrollo de la apertura comercial y globalización comercial que tuvo el país.

Las aportaciones de estos cuestionamientos a la investigación científica no sólo se centrarán en el ámbito administrativo y de operación portuaria, sino también en la discusión económica, en especial en la aplicación de políticas públicas y su efecto en el desarrollo regional costero del país. También existen aportaciones sustantivas en el análisis jurídico-administrativo que enmarcan la normatividad de operaciones de los recintos portuarios industriales, ya que se pretende esclarecer los efectos en el desarrollo urbano-regional como consecuencia en la aplicación de las reformas administrativas llevadas a cabo en el periodo 1970-2005.

Para dar respuesta a las preguntas centrales del texto, el autor desarrolla ampliamente con datos duros y cifras la evolución histórica del enclave dual de Tampico y Altamira, las llamadas ciudades gemelas. El análisis se desarrolla a lo largo de cinco capítulos, con una descripción metodológicamente bien estructurada. Cabe mencionar que este concepto de ciudades gemelas lo han utilizado indistintamente autores que explican la dualidad funcional entre dos ámbitos urbanos o metropolitanos, algunos referidos inclusive a localizaciones transfronterizas (Alegría; 2009) cuyo contexto refiere a un complejo urbano dual con funcionalidad integral, económica o social.

Los dos primeros capítulos tratan aspectos similares, ambos nos introducen a entender aspectos contextuales de operación y administración, así como los que circunscriben el contexto territorial. Especial interés pone el autor en describir las circunstancias económicas y de competencia mundial que se generan como producto de los procesos de la globalización comercial, la cual se identifica sobre todo por el incremento en los movimientos masivos de las mercancías internacionales a través de los puertos industriales más importantes del orbe. México no es la excepción de ello.

El proceso de globalización el autor lo evalúa y explica claramente en los campos de las oportunidades y ventajas comparativas que conlleva el estar inserto en el concierto mundial de países en vías de desarrollo, así como la circulación de mercancías, redistribución regional de la mano de obra y la construcción y adecuación de las infraestructuras y equipamientos regionales.

Sin embargo, con acierto, el autor comenta los límites de esta inserción mundial en un mercado tan globalizado y tan competido, ausente en muchos casos de reglas claras. Se mencionan también las ventajas de localización que tiene los puertos mexicanos a lo largo de las costas del Pacífico y del Golfo de México. El referente conceptual y estadístico lo retoma de Carlos Martner (Martner; 1996), quien ha profundizado en el 
campo de la globalización comercial y el papel de los puertos industriales en México.

El establecimiento de redes transnacionales de puertos industriales receptores de movimientos mercantiles es una de las paradojas que desentraña José Zorrilla. En su evaluación analiza metodológicamente el significado de puerto industrial, el concepto del llamado contenedor, así como sus modificaciones técnicas con el paso del tiempo. También explica técnicamente la importancia de la estandarización mundial en pesos y medidas en los contenedores y la forma en que estos se acomodan y distribuyen en los puertos industriales. También se describe ampliamente lo que esto significa en tiempos y movimientos, así como sus efectos en la economía local, nacional y mundial. Este salto cualitativo en la optimización de las operaciones representa la diferencia entre estar o no en la esfera de la competencia global.

De esta manera, el autor aproxima una explicación profunda en la operación de los puertos sujetos de estudio, los tonelajes de mercancías movidas por tipo de mercancía, su almacenamiento y destino, la competencia directa con puertos industriales localizados en Estados Unidos, Asia, Latinoamérica y Europa.

Un aspecto importante del trabajo de Zorrilla González es el referente a la vinculación de los puertos en el espacio y el tiempo, en cuanto a su situación geográfica, su evolución tecnológica y nivel cultural. El autor denomina a este fenómeno puntos de ruptura pero a la vez lugares de encuentro, así, los puertos se constituyen en lugares de interacciones internas y externas, intersecciones de rutas comerciales y zonas de confluencia e intercambio de ideas. Por ello la operación de los puertos industriales también se convierte en promoción de infraestructura orientada a las tecnologías de la información, a generación de fuentes indirectas de trabajo, producto de su gran efecto multiplicador y con ello de bienestar social.

En otra esfera de análisis teórico, diversos autores ya habrían estudiado el papel de los llamados enclaves de producción, teoría de los polos de crecimiento o de desarrollo, entendida como un conjunto industrial imbricado en torno a una dinámica industrial central a través de una serie de encadenamientos de insumo-producto en un mercado determinado (Perroux et al., 1973).

Todos los cambios económicos que surgen de la vinculación con el comercio mundial que tienen las economías emergentes, como la de México, pueden venir acompañados de ajustes estructurales e institucionales para su funcionamiento. Zorrilla propone a la reforma portuaria de 1993 como piedra angular del cambio, mismo que inicio en 1991, año 
en que el gobierno federal requisa el puerto de Veracruz y asume temporalmente la administración y operación del mismo.

Con esta intervención, o golpe de timón, llevada a cabo por el gobierno federal, se liquida una de las tantas empresas paraestatales creadas en el sexenio de Luis Echeverría en el año 1973. Muchos de los autores y analistas contemporáneos, entre ellos Zorrilla González, consideraron positivos los efectos inmediatos de esta medida. Sólo un año después dicha requisa mostró sus efectos positivos, la operación de este puerto se incrementó $48 \%$, los salarios se elevaron $200 \%$ para el trabajador de muelle y las tarifas disminuyeron $30 \%$. Al parecer fue todo un éxito.

La reforma a la Ley de Puertos contempló ampliamente toda la plataforma justificativa para la nueva forma de operación de los recintos portuarios, siendo el capítulo referente a la administración portuaria integral (API's) de esta reforma la que mayor efecto tuvo en la operación portuaria del país. En este sentido, la nueva Ley de Puertos, según Zorrilla González, fue todo un éxito para el caso de Veracruz. En la actualidad se encuentran bajo este régimen 25 API's, 16 de la cuales son de naturaleza federal a cargo de la Coordinación General de Puertos y Marina Mercante; dos de tipo paraestatal que dependen de Fonatur; cinco estatales y cinco bajo la coordinación de municipios.

Otro aspecto importante del trabajo de investigación elaborado por Zorrilla fue el análisis crítico de los pro y contras de la operación dentro de las API's, poniendo en la mesa de debate las características de participación extranjera en la inversión de los recintos portuarios, misma que hoy día se rige por la Ley General de Sociedades Mercantiles. La concesión de estas administraciones portuarias es el tema actual en la operación exitosa o, en su caso, de su bajo rendimiento.

En general, el segundo capítulo concluye con reflexiones en torno a cuatro aspectos: Primero, la reforma de 1993 permitió establecer lo que llamaríamos reglas claras de operación, que disminuyen la incertidumbre entre los transportistas y usuarios comerciales. Segundo, se establecieron las reglas claras y transparentes en torno a la aplicación de la ley, así como las normas y procedimientos eficientes para cada uno de los puertos comerciales. Tercero, se creó un esquema de certidumbre en el marco de la globalización, los sistemas de información necesarios y los mecanismos de distribución eficientes. Y por último, se estableció un proceso de adaptabilidad donde los puertos responden rápidamente a la eventualidad de los cambios. Douglas North (North y Weingast, 1989) se encuentra presente a lo largo de la obra de Zorrilla González, su análisis nos deja entrever su dominio en el tema y la facilidad para vincular este nivel de abstracción teórica con la realidad analizada en los puertos industriales en México. 
El tercer capítulo realmente no tiene desperdicio, en él se vierte el análisis histórico y por demás sustantivo del trabajo de investigación. Los puertos de Tampico y Altamira se decriben de forma detallada, sus similitudes y diferencias, así como las perspectivas de crecimiento de ambos.

En esta sección podemos observar y entender, con datos duros, lo que sucede en cada uno de los enclaves industriales. La evolución histórica del viejo puerto de Tampico dedicado sobre todo al transporte de productos mineros y de la industria extractiva. La creación de Altamira se describe muy atractiva, ya que este puerto fue creado por el extinto Fondo Nacional para los Desarrollos Portuarios (Fondeport) con la intención de integrar un desarrollo regional más allá del manejo de entrada y salida de mercancías con destino internacional. El ejemplo de ello es que son de los pocos recintos portuarios que juntaron reservas territoriales acordes al potencial del desarrollo urbano de las zonas y del ámbito regional. El puerto de Altamira basa su operación en el ramo de la petroquímica tanto estatal como privada.

También Carlos Martner (1996) es una referencia continua del autor, recurre a sus datos y cifras en el manejo y volúmenes de carga de los puertos industriales del país, así como del análisis explicativo de los efectos regionales y zonales de la creación del enclave industrial. Este capítulo concluye con la mención de la articulación del sistema Tampico-Altamira, misma que permite tener un sistema dual con interdependencias y especialidades individuales en sus procesos, que permite a su vez esta consolidación portuaria única en costas mexicanas.

En el capítulo cuarto se aborda esta dualidad desde una óptica comparativa, el objetivo que el autor reseña es el de poder distinguir e identificar las respectivas vocaciones de cada uno de los puertos, así como identificar cuál es el destino de la carga operada, sus importaciones y exportaciones, así como la definición y especialización del tipo de carga. Posteriormente nos lleva a entender las diferentes etapas que ha experimentado el complejo portuario en las últimas tres décadas, así como la exposición de cifras del desempeño en la operación con las características del modelo económico que el país mantenía en ese tiempo.

Resulta atractiva la vinculación que se realiza en torno a la etapa económica del llamado desarrollo estabilizador, donde la sustitución de importaciones y la política proteccionista genera un sistema complejo de permisos y licencias. En esta fase del desarrollo del país Tampico experimenta una expansión moderada, con una tasa de crecimiento de $3.4 \%$ anual, la cual asciende en 2005 a 5\%, debido principalmente a la petrolización de la economía mexicana.

Cabe mencionar que el desarrollo estabilizador es el modelo económico aplicado en la economía mexicana hacia la década de los cincuenta y 
finales de los setenta, modelo que facilitaba mantener en equilibrio indicadores macroeconómicos como la inflación, el déficit interno y la balanza comercial (Cordera y Ziccardi, 2000).

Alternativamente, la construcción del Puerto de Altamira ofrece una opción real a la saturación de las operaciones de Tampico, pero también permite la vinculación entre exportaciones e importaciones a un mercado internacional más dinámico, en el plano de la liberación económica y la globalización comercial. Modelos económicos que impusieron circunstancias contradictorias en la toma de decisiones en la política general de desarrollo de México.

Esta dualidad de las operaciones, menciona el autor, tuvo como primera consecuencia el manejo de los volúmenes de operaciones portuarias. El efecto en Tampico fue su disminución operativa hacia los años ochenta. El total de carga descendió de 18\% (1970-1979) del total que se movía en los puertos mexicanos a 3.4\% (2000-2005). De esta forma Tampico cede su importancia industrial a los puertos de Altamira y Veracruz.

A finales del año 2005 la actividad de cabotaje vuelve a activarse en Tampico sobre todo por los derivados del petróleo, como el crudo, diesel y gasolina, especialización que es atraída por el crecimiento importante que produce Altamira. Zorrilla González desliza una conclusión interesante: Altamira mantiene una historia opuesta a Tampico, su crecimiento va aparejado al decrecimiento de este último. La tasa de crecimiento anual de Altamira es sostenida, muy por arriba del nacional o regional.

En el análisis comparativo descubrimos la dualidad urbana producida por los enclaves industriales gemelos, aunque el autor no aborda como tema central el proceso de desarrollo urbano o metropolitano, propone las bases para una futura reflexión, principalmente ligada a la dinámica regional producida en la costa del Golfo de México.

Existen buenas investigaciones que analizan la evolución urbana y metropolitana, así como el efecto territorial y social a partir de la firma del tLCan (Ramírez y Pradilla, 1993). Los enclaves industriales serían piezas territoriales importantes para que inviertan, sobre todo, firmas norteamericanas. Las ciudades costeras del Golfo de México con potencial industrial, de producción y almacenamiento jugarían un papel clave, ya que ahí se concentrarían, además de las inversiones, procesos acelerados de urbanización y pauperización.

En el último capítulo de la obra se reflexiona nuevamente sobre los efectos en la organización del sistema portuario Tampico-Altamira, reiteración que pareciera importante toda vez que analiza los procesos de adaptabilidad que deben tener las administraciones portuarias para poder subsistir ante la feroz competencia internacional. 
Se reitera la importancia que ha tenido la reforma de la Ley de Puertos de 1993 y su efecto en la organización gremial, en especial la que comandaba el Gremio Unido de Alijadores, el cual se obligó a firmar un contrato de cesión parcial de derechos. De esta forma el sindicato se transformó de una organización laboral especializada a una sociedad mercantil. La cuestión ahora es saber cuáles fueron los efectos reales de esta transformación en el entorno social y político, y si esto trajo como consecuencia un beneficio a los trabajadores y no sólo a las operaciones portuarias. Análisis y respuestas que no se incluyen en el estudio, pero que se abren como futuras líneas de investigación.

En el balance general de la obra, ésta resulta de suma importancia para entender a profundidad el comportamiento de las operaciones en los puertos industriales de México, asimismo invita a la reflexión profunda que Zorrilla expone con un vasto acervo de datos, expresados en tablas y gráficas comparativas muy extensas del acontecer entre la dualidad portuaria de Tampico y Altamira.

La reflexión nos recuerda la vocación personal y profesional del autor, cuya formación está en el estudio del derecho, una maestría en derecho fiscal, ambas llevadas a cabo en la Universidad Regiomontana. Las diversas especialidades y líneas de estudio posteriores a su formación tanto en el país como en el extranjero lo llevaron a obtener el grado de doctor en El Colegio Mexiquense en ciencias sociales. La vocación académica también lo consolida como investigador y fundador de El Colegio de Tamaulipas en las áreas del desarrollo regional y economía marítima.

Un reconocimiento al esfuerzo académico de la obra de José Zorrilla González que se cristaliza en este libro tan importante para la reflexión de los puertos industriales del sur de Tamaulipas.

\section{Bibliografía}

Alegría Olazábal, Tito (2009), Metrópolis transfronteriza. Revisión de la hipótesis y evidencias de Tijuana, México y San Diego, Estados Unidos, Miguel Ángel Porrúa-El Colegio de la Frontera Norte, México.

Cordera, Rolando y Alicia Ziccardi (2000), Las políticas sociales de México a fin del milenio: descentralización, diseño y gestión, Universidad Nacional Autónoma de México, México.

Martner, Carlos (1996), "Transporte y concentración territorial en América Latina: tendencias recientes", Ciudad y Territorio: Estudios Territoriales, 110, Ministerio de Fomento, Madrid. 
Perroux, François, John Friedman y Jan Tinbergen (1973), Los polos de desarrollo y la planificación nacional, urbana y regional, Nueva Visión, Buenos Aires.

Ramírez, Blanca y Emilio Pradilla (1993) "El tratado norteamericano de libre comercio y la integración territorial de México a Estados Unidos", Revista Interamericana de Planificación, xxvI (103), sIAP, México, pp. 19-54.

Recibido: 10 de enero de 2012. Reenviado: 20 de febrero de 2012. Aceptado: 12 de octubre de 2012.

Rafael Valdivia-López Universidad Autónoma Benito Juárez, Oaxaca Correo-e: rvlcon@gmail.com

Rafael Valdivia-López. Es doctor en ciencias sociales por El Colegio Mexiquense con la investigación "Modelo de precios hedónicos para la vivienda nueva en la ZMvM". Llevó a cabo el curso Planeación y gestión urbana, en el Instituto de Estudios de Administración Local, Madrid, España; asimismo ha hecho las siguientes estancias doctorales: en el Departamento de Análisis Geográfico Regional y Geografía Física en la Universidad Complutense de Madrid y en la Facultad de Ciencias Económicas de la Universidad Castilla-La Mancha, España. Es profesor-investigador en el Instituto de Investigaciones Sociales (IIs) de la Universidad Autónoma Benito Juárez (UABJo). En el sector público ha ocupado diversos cargos, entre otros, en la Dirección General de Verificación del Ordenamiento Ecológico e Impacto Ambiental, en la Profepa (19972001). Entre sus publicaciones destacan: "Modelos IDw para la toma de decisiones en materia de construcción de vivienda y adquisición de suelo urbano", en Diego Alfonso E. (coord.), Sistemas de información geográfica aplicados a estudios urbanos, Lincoln Institute of Land Policy, Massachussets, pp. 121-131 (2006); Un modelo de econometría espacial "precios hedónicos" para desarrollos habitacionales, Instituto de Pesquisa Económica Aplicada (IPEA), Sao Paulo, Brasil (2010); "Monitoreo para la prevención de incendios forestales, utilizando técnicas de percepción remota y sIG", en Francisco Garfias (coord.), Incendios forestales y agropecuarios, UNAMIPN-Semarnat, México, pp. 37-52 (1998). 